
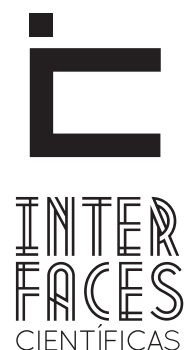

EDUCAÇÃO

ISSN IMPRESSO 2316-333X

ISSN ELETRÔNICO 2316-3828

PARTE 2 - FORMAÇÃO E ATUAÇÃO PROFISSIONAL DA EDUCAÇÃO SOCIAL

\title{
UNIVERSIDAD Y FORMACIÓN DE PROFESIONALES DE LA EDUCACIÓN SOCIAL
}

\section{RESUMEN}

El presente artículo se centra en la Pedagogía Social como ciencia que fundamenta una profesión que es la Educación Social. La universidad debe garantizar el desarrollo de políticas sociales que garanticen cubrir las necesidades educativas de la ciudadanía. Desde las universidades españolas se oferta el Grado en Educación Social, título adaptado al Espacio Europeo de Educación Superior. El ejercicio de los educadores y las educadoras sociales conlleva la idea de que la finalidad de sus acciones es el servicio directo a las personas, de manera individual y/o colectiva. Desde la universidad se forma a estos profesionales para la acción e intervención socioeducativa desde una perspectiva del aprendizaje a lo largo de la vida, paradigma que se debe tener presente en todos los ámbitos de la educación social.

\section{PALABRAS CLAVE}

Universidad. Pedagogía Social. Educación Social. aprendizaje a lo largo de la vida. 


\section{RESUMO}

0 presente artigo se centra na Pedagogia Social como ciência que fundamenta uma profissão que é a Educação Social. A universidade deve fundamentar o desenvolvimento de políticas sociais que garantam corresponder às necessidades educativas da cidadania. Nas universidades espanholas se oferta a graduação em Educação Social, título adaptado ao Espaço Europeu de Educação Superior. 0 exercício dos educadores e educadoras sociais exprime a ideia de que a finalidade de suas ações é o serviço direto às pessoas, de ma- neira individual e coletiva. Desde a universidade, estes profissionais são formados para a ação e intervenção socioducativa a partir de uma perspectiva de aprendizagem ao longo da vida, paradigma que se deve ter presente em todos os âmbitos da Educação Social.

\section{PALAVRAS-CHAVE}

Universidade. Pedagogia Social. Educação Social. Aprendizagem ao Longo da Vida.

\section{ABSTRACT}

This article haves focus on Social Pedagogy as a science that founds a profession that is Social Education. The university should support the development of social policies that ensure correspond to educational needs of citizenship. In spanish universities is offered a degree in Social Education, academic title adapted to the European Higher Education Area. The exercise of social educators expresses the idea that the purpose of their actions is the direct service to people, individually and collectively. From the university, these professio- nals are trained for action and intervention social and educational from a perspective of lifelong learning, paradigm that must have present in all areas of Social Education.

\section{KEYWORDS}

University. Social Pedagogy. Social Education. Lifelong Learning. 


\section{POLÍTICAS SOCIALES Y UNIVERSIDAD}

De acuerdo con la Organización de las Naciones Unidas (ONU) la política social es un instrumento que utilizan los gobiernos para regular y complementar las instituciones del mercado y las estructuras sociales. Con frecuencia se define en términos de servicios sociales como la educación, la salud, o la seguridad social. Sin embargo, incluye mucho más: distribución, protección y justicia social. La política social consiste en situar a la ciudadanía en el núcleo de las políticas públicas, ya no mediante el suministro de asistencia social residual, sino incorporando sus necesidades y voz en todos los sectores, para que pueden superar el círculo vicioso de la pobreza y el atraso, y crear un meridiano virtuoso en el que el desarrollo humano y el empleo generen una mayor demanda interna y crecimiento económico (ORTIZ, 2007).

Las políticas sociales, como parte de las políticas públicas, proporcionan un conjunto de directrices, orientaciones y criterios orientados a la preservación y elevación del bienestar social, procurando que los beneficios del desarrollo alcancen a todas las capas de la sociedad con la mayor equidad (VITERI DÍAZ, 2007). A través de ellas, se interviene en la realidad aunando y coordinando recursos con el fin de aumentar el bienestar de la población, por lo que constituyen una parte esencial de cualquier Estrategia Nacional de Desarrollo, para lograr el crecimiento económico, la equidad social y reducir las situaciones de desventaja social y económica, y por consiguiente la pobreza y la exclusión.

A las políticas sociales con el paso de los tiempos se les ha fijado distintos objetivos, como la igualdad de oportunidades y de género, la superación de la pobreza, la atención a las personas mayores, la prevención y tratamiento del consumo de drogas, etc., para conseguir mayores cotas en la calidad de vida de la población. Los profesionales saben que para alcanzar estos objetivos son necesarias estra- tegias que redistribuyan recursos y bienes de modo más equitativo, ya sea poniendo en marcha "políticas sociales clásicas" (las propias de la sociedad de bienestar: asistencia sanitaria, vivienda, pensiones, educación...) o impulsando "políticas de integración social" dirigidas a colectivos que presentan problemas específicos, como los relacionados con la discriminación de las mujeres y la violencia en todas las etapas de la vida y contextos, la atención a la infancia, adolescencia y mayores, o la intervención respecto a las drogodependencias. Políticas sociales que se fundamentan en la Declaración Universal de los Derechos Humanos (ONU, 1948), por lo que constituyen parte de las funciones primarias del Estado.

\subsection{LA UNIVERSIDAD COMO SERVICIO PÚBLICO}

La relación entre demanda social y oferta universitaria no es exclusiva de la universidad contemporánea, pues se remonta a sus orígenes (TORSTENDAHL, 1996; GINER DE LOS RÍOS, 1919; ORTEGA y GASSET, 1930). Las titulaciones universitarias están destinadas a cubrir una serie de necesidades educativas y, sobre todo, unas exigencias de tipo social, cultural y económico; de ahí que cuando el alumnado finaliza sus estudios universitarios se asuma, con propiedad, que se encuentra capacitado para enfrentarse al mercado laboral y poner en práctica los conocimientos, habilidades, actitudes y valores adquiridos durante su etapa de formación. Esta congruencia de la universidad con las exigencias emergentes, de las sociedades actuales, forma parte de los procesos de modernización que las universidades deben encarar para su desarrollo en el mundo.

Los cambios sociales han ido promoviendo reformas en la Educación Superior, sin embargo, la esencia de la universidad se mantiene, su razón de ser trasciende toda época, lugar o circunstancia social, y su labor docente e investigadora en la formación de profesionales constituye un referente. 
Ortega y Gasset (1930) en su artícuo “Misión de la universidad" reflexionan sobre la enseñanza superior ofrecida en la Universidad, para él consistía en la enseñanza de las profesiones intelectuales; en la investigación científica y la preparación de futuros investigadores, y, en la enseñanza de la cultura. Sostiene que la sociedad necesita buenos profesionales -jueces, médicos, ingenieros-, pero reclama que estos profesionales sean capaces de vivir e influir vitalmente en su época. Por consiguiente, en la sociedad actual es imprescindible la formación de profesionales sobre los problemas sociales que aquejan a la población, para contribuir al cumplimiento de los derechos humano y conseguir el compromiso institucional de mejorar la calidad de vida de la ciudadanía.

Junto a la doble misión tradicional de enseñanza e investigación, la Universidad desempeña un papel creciente en los "procesos de innovación", en la "cohesión social" y en materia de desarrollo económico y social (BARRAYCOA; LASAGA, 2010). Las universidades, además de un motor para el avance del conocimiento, deben ser un motor para el desarrollo social y económico del país, promoviendo la transferencia a la sociedad de los resultados de su investigación, lo que contribuirá a la consecución de un mayor grado de bienestar de la ciudadanía.

La formación universitaria está vinculada con el desarrollo profesional en contextos laborales, pero sin olvidar el desarrollo del pensamiento crítico, dado que el progreso de un país está no sólo en tener profesionales técnicamente competentes, sino en que, además, sean capaces de pensar de manera autónoma y de acuerdo con los principios basados en los Derechos Humanos. La cuestión reside en preguntarse por el tipo de personas que se pretende formar desde la educación y la Pedagogía Social, así como en su transcendencia para intervenir ante numerosas cuestiones sociales (personas adultas y mayores, género, exclusión social, pobreza, consumo de drogas etc.) desde una profesión como es la Educación Social.

\section{PEDAGOGía SOCIAL Y EDUCACIÓN SOCIAL}

Como han puesto de relieve diferentes autores (QUINTANA, 1984, 1986; SÁEZ, 1986, 2007; PETRUS, 1989; PÉREZ SERRANO, 2003; CARIDE, 2005), a comienzos de los años 1980 tuvo lugar el resurgimiento de la Pedagogía Social, iniciándose un camino que llega a nuestros días con grandes aportaciones a su campo de conocimiento teórico y a las cuestiones sociales que investiga, desde una perspectiva educativa (personas mayores, género, infancia y adolescencia, exclusión, maltrato, violencia, delincuencia, drogodependencias, ocio y tiempo libre, etc.); sus contribuciones constituyen el fundamento de la actual titulación en Educación Social, de la formación de su alumnado y de su profesionalización. Profesionalización entendida como una acción educativa fundamentada, reflexiva y planificada.

Podemos afirmar que actualmente existe acuerdo entre los miembros de la comunidad científica de Pedagogía Social (QUINTANA, 1984, 1986; SÁEZ, 1986, 2007; ORTEGA, 1999; PÉREZ SERRANO, 2003; MERINO, 1989; CARIDE, 2005; PETRUS, 1989; NUÑEZ, 1999) a la hora de entender la Pedagogía Social como ciencia de la Educación Social, es decir, como un campo de saber o "un marco teórico desde el cual podemos pensar las cuestiones que atañen a la educación social" (NÚÑEZ, 1999, p. 32), que es su objeto de estudio. En este sentido, Ortega (1999a, p. 13) al referirse a la praxis de la educación social, reclama "una disciplina científica que teorice y conceptualice, que investigue, organice, recopile y sistematice los conocimientos relativos a esa acción o práctica profesional no hay ciencia o disciplina sin conceptualización. Sin conceptos claros no es posible actuar.

Nadie puede conformar o actualizar o reformular una práctica si no tiene los conceptos claros, los cuales tienen que estar asentados en una teoría científica lo más fundada posible en la investigación, en la reflexión sistemática o en la construcción sincrética 
con sus propios encuentros y con los aportes de otras ciencias relacionadas con esa disciplina, en nuestro caso, expresamente orientada a la educación social”, que al materializarse en contextos complejos lleva consigo la transformación, en la medida de lo posible, de la realidad mediante las prácticas educativas (ETXEBERRÍA, 1989), realizadas por profesionales de la Educación Social con las personas destinatarias de las mismas, como en el caso de la intervención en cuestiones relacionadas con personas adultas y mayores, infancia y juventud, exclusión, pobreza, violencia, desigualdad, género, drogodependencias, etc.

En suma, la educación social es una práctica educativa construida y materializada por los educadores y educadoras sociales, en organizaciones e instituciones donde se demanda su acción profesional. Y, es, desde la Pedagogía Social desde donde se investiga y fundamenta esta práctica educativa. Pero como ciencia está siempre en permanente evolución, es un saber inacabado, en constante estado de revisión y discusión. Parafraseando a Morin (2001) diríamos que es una isla de certeza en un océano de incertidumbres.

La comunidad científica de la Pedagogía Social coincide en señalar que su objeto de estudio es siempre la educación social, aunque desde una concepción clásica de la epistemología, suele ser habitual distinguir entre el objeto formal y material. La diferencia estriba en que como objeto formal es una abstracción, viene dado por el tipo de conocimiento que utiliza y la perspectiva desde la que estudia su objeto material, así como por la realidad que analiza; $y$, como objeto material es una práctica educativa recreada en contextos y escenarios de actuación social y educativa, es decir, en aquellos ámbitos de preocupación (como en los citados anteriormente y la acción educativa llevada a cabo) en los que intervienen los educadores y educadoras sociales, poniendo en juego, mediante sus prácticas educativas, sus competencias personales y profesionales, de acuerdo con las necesidades y demandas de la comunidad a la que pertenecen. actualmente, estas prácticas son abundantes por la variedad y complejidad de los problemas sociales; además, se diseñan y realizan en multitud de organizaciones de titularidad pública o privada, y son construidas en los contextos requeridos, involucrando tanto a los profesionales de la Educación Social, como a otros que trabajan con ellos, para promover situaciones de relación educativa en la que se produzca la transmisión de conocimientos, la mediación cultural y educativa, y la generación de un clima de comunicación que promueva la reflexión y la toma de decisiones autónoma, responsable y libre (BAS-PEÑA, CAMPILLO Y SÁEZ, 2010).

Quintana (1984) en su obra “Pedagogía Social” recoge entre las Áreas de intervención pedagógica la ayuda a la familia y a sus miembros sujetos a proble $\neg$ mas existenciales (drogodependencia, tercera edad: integración del anciano, y la marginación social de la mujer. Dos años más tarde, el profesor Quintana (1986) en su artículo "La profesionalización de la Pedagogía Social” hace referencia a su dimensión científica y profesionalizadora, afirma que ésta no es sólo una especialidad profesional-pedagógica, sino un conjunto de ellas, las identificadas hasta el momento, que considera que son las más importantes, ratificando las propuestas en 1984.

Merino (1989) nos habla de matriz epistemológica y fundamenta, la "inadaptación y delincuencia juvenil" como objeto de la Pedagogía Social, porque ésta se ocupa de la educación social dentro y fuera de la escuela. Propuestas y ámbitos corroborados, años después, por Petrus (1989, p. 22) en el Congreso organizado por el Ministerio de Educación y Ciencia sobre "La Educación Social en España”, años previos a la creación de la diplomatura en Educación Social (1991), que contemplará tres grandes ámbitos en los que se integraran las demandas expresadas: Educación Especializada; Educación de Adultos; Animación Sociocultural.

En suma, la educación social es una práctica educativa construida y materializada por quienes se dedi- 
can profesionalmente a la Educación Social. El campo de conocimiento de la Pedagogía Social investiga y fundamenta esta práctica educativa, por lo que no puede plantearse su objeto material de modo abstracto, sin hacer referencia a los espacios o equipamientos donde se llevan a cabo sus prácticas educativas y profesionales. De esta forma se vincula la teoría y la práctica que estudia, estableciendo una relación dialéctica, en donde la teoría tiene que dar razón de los espacios/ámbitos en los que se materializan esas prácticas, creando un continuum entre teoría y práctica. Además, unir la investigación al análisis de la acción práctica, pensando en construir conocimiento teórico, es potenciar la relación entre teoría y práctica para trabajar en la clarificación de esta dicotomía, tan significativa en ámbitos profesionales, porque "proporciona un medio para trabajar que vincula la teoría y la práctica en un todo único: ideas en acción" (KEMMIS; MCTAGGART, 1992, p. 10).

En definitiva, la Pedagogía Social como ciencia y matriz disciplinar fundamenta la Educación Social como titulación, profesión social y educativa, y práctica educativa.

\section{LA EDUCACIÓN SOCIAL}

La Asociación Estatal de Educadores Sociales (ASEDES, 2007) define la Educación Social desde un doble eje: como derecho a la ciudadanía y como profesión de carácter pedagógico; suscribe que es "el derecho de la ciudadanía que se concreta en el reconocimiento de una profesión de carácter pedagógico, generadora de contextos educativos y acciones mediadoras y formativas, que son ámbito de competencia profesional del educador social y que posibilitan: - la incorporación de los sujetos de la educación a la diversidad de las redes sociales, tanto en lo concerniente al desarrollo de la sociabilidad como a las posibilidades de circulación social;- la promoción cultural y social, entendida como apertura a nuevas posibilidades de adquisición de bienes culturales que amplíen las perspectivas educativas, laborales, de ocio y participación social”.

La educación social presenta diversas acepciones (FERMOSO, 2003) la entiende como prevención y control social, como parte integrante de la educación total de la persona y como adoctrinamiento político. Este autor la define como sociabilidad e indica que

\begin{abstract}
Es el resultado o producto del proceso de socialización, equivalente o traducible en un conjunto de habilidades desarrolladas por el aprendizaje, que capacitan al hombre para convivir con los demás y adaptarse al estilo de vida dominante en la sociedad y cultura a la que se pertenece, sin perder la identidad personal, aceptando y cumpliendo, al menos, sus (de la sociedad y de la cultura) exigencias mínimas. (FERMOSO, 2003, p. 66).
\end{abstract}

Coexisten planteamientos acerca de la Educación Social, principalmente en dos direcciones (MOYANO, 2007, p. 53):

- $\quad$ Orientada en su mayor parte a la ayuda social de los individuos, fijada al adjetivo social y con tendencias a pesar de lo educativo tan solo como elemento agregado a un trabajo social compensatorio más amplio que incluiría cuestiones relacionadas con lo emocional, la contención y la prevención social. En esta línea se sitúan algunas propuestas vehiculizadas a partir de la necesidad de empoderamiento del individuo o la capacitación para la resiliencia.

- Plantea lo educativo como constructivo del concepto, que apuesta por la educación como eje principal de las prácticas que se desarrollan en este campo. Es decir, una propuesta de carácter principalmente pedagógico que permita la articulación de los individuos con las exigencias sociales de época. La educación, en este sentido, se convierte en el pivote configurador de la Educación Social y no un elemento más.

El Real Decreto 1420/1991 publicado en el BOE de 10 octubre por el que se creaba el título oficial universitario de Diplomado en Educación Social, afianza 
la constitución de sectores profesionales: educación especializada, animación sociocultural y educación de adultos. Textualmente se puede leer: "las enseñanzas deberán orientarse a la formación de un educador en campos de la educación no formal, educación de adultos (incluidos los de tercera edad), inserción social de personas desadaptadas y minusválidos, así como de la acción socioeducativa”. Trata de dar respuesta a organizaciones y entidades con larga trayectoria en estos campos prácticos.

En Europa estamos sumergidos en cambios estructurales a nivel universitario. Con la mundialización de los estudios superiores y la creación del Espacio Europeo de Enseñanza Superior, que pone en marcha una reforma estructural y curricular de los planes de estudios de las universidades de los estados miembros, se publica, en el año 2004, el Libro Blanco del Título de Grado en Pedagogía y Educación Social, donde se enfatiza el dinamismo de la sociedad del conocimiento y los nuevos desafíos que se le plantean a la educación como ámbito profesional y de estudio. En palabras de Echeverría (2009, p. 35):

\begin{abstract}
Los cambios sociales que se han producido en Europa $y$ en el conjunto de las sociedades occidentales han sido muy profundos y han afectado intensamente al mercado de trabajo, la economía y estilos de vida de los ciudadanos. La amplitud y profundidad de estas transformaciones culturales, sociales y económicas es de tal dimensión que, los expertos consideran que el conocimiento y la información han pasado a ser factores fundamentales para gestionar las diferencias entre personas, organizaciones y países. Estos cambios demandan en las organizaciones e instituciones una renovada configuración de la profesionalidad.
\end{abstract}

Las transformaciones acaecidas, en las últimas décadas, en el ámbito universitario y en la sociedad hacen que la Educación Social se centre fundamentalmente en problemas humano-sociales, en contacto directo con la realidad de las personas. Es tarea de la Educación Social el enriquecimiento, la forja de la persona que vive en un momento dado, con una cultura propia y en una sociedad concreta, en la cual es necesaria la comunicación interpersonal como forma de vinculación social. Dentro de este proceso, el educador o educadora social es un profesional que realiza una acción socioeducativa con personas y/o grupos, desde una línea normalizada o especializada; con objeto de generar cambios para la mejora y transformación social. Promueve la educación a lo largo de toda la vida, en todas sus dimensiones, mediante la participación y el desarrollo del espíritu crítico. Su labor se desarrolla en contextos sociocomunitarios, principalmente en el ámbito no formal.

Conviene señalar la importancia social y profesional de promover y potenciar la formación en cuestiones sociales concretas sobre personas mayores, infancia y juventud, género y violencia de género, drogodependencias, pobreza,.. en el Grado en Educación Social, de manera que capacite a quienes en el futuro ejerzan esta profesión, para que puedan dar respuestas educativas a las problemáticas indicadas (BAS-PEÑA, PÉREZ-DE-GUZMÁN Y VARGAS-VERGARA, 2014).

\subsection{EDUCACIÓN SOCIAL: PROFESIÓN SOCIAL Y EDUCATIVA}

La revisión de la literatura especializada muestra las dificultades que existen para llegar a un consenso sobre el concepto de profesión, porque el término profesión es polisémico, se puede estudiar en diferentes campos semánticos, asociado a diversos usos, y de acuerdo con modelos teóricos distintos. Desde finales del siglo XIX y principios del siglo XX son numerosas las definiciones de profesión, realizadas, principalmente, desde el campo de la sociología o de la historia de las profesiones.

Freidson (2001) manifiesta que por lo menos desde Flexner (1915), gran parte del debate se ha centrado en cómo se deberían definir las profesiones, aunque aún no se ha logrado el consenso, es más, apenas se ha evolucionado desde los primeros debates. Actualmente "no existe una receta universal 
de cómo ser un profesional; no hay, como postula el taylorismo, un one best way para organizar el trabajo profesional y tampoco hay una definición única de lo que es un grupo profesional” (PANAIA, 2008, p. 10), y dado que su uso varía sustancial, lógica y conceptualmente (FREIDSON, 1977), algunos estudiosos manifiestan estar en desacuerdo en la práctica misma de buscar una definición.

Sin embargo, Freidson (2001, p. 30) piensa que “para pensar clara y sistemáticamente sobre cualquier cosa, uno debe delimitar la materia por abordar mediante el análisis empírico e intelectual. No podemos elaborar una teoría si no estamos seguros sobre lo que tratamos", pero es consciente de las dificultades para conseguir el acuerdo, principalmente por al carácter histórico y concreto del concepto, así como por las diferentes perspectivas desde las cuales se puede ver legítimamente y puede tener sentido, así como por la gran heterogeneidad de las prácticas profesionales. Todo ello, justifica el fuerte pluralismo encontrado en la teoría sociológica donde cada corriente de pensamiento desarrolla un modelo privilegiando sobre las profesiones, su estructura, su dinámica, su función y sus efectos.

La preocupación por las profesiones y el estudio de sus respectivos procesos profesionalizadores llega a las universidades ya avanzado el siglo pasado, y sucede lo mismo con los trabajos sobre la profesionalización del profesorado universitario. Diferentes autores (LARSON, 1977/1979; JOHNSON, FREIDSON, 1977, 2001; SÁEZ, 2007) exponen que las profesiones, además de cumplir funciones económicas, actúan como intermediarias entre el sistema educativo y el mercado, ya que las competencias adquiridas durante el proceso de formación se materializan en la acción, en los espacios laborales ofertados, como consecuencia de las políticas públicas diseñadas por el Estado, como hemos indicado anteriormente.

En el caso de las profesiones sociales, la sociología de las profesiones, no se interesó por ellas hasta después de la Segunda Guerra Mundial, aunque en España no tuvo lugar hasta finales de los años setenta. Actualmente, algunas de ellas ya se han confirmado como profesiones, tal es el caso de la Educación Social. Por otra parte, las nuevas realidades y conflictos sociales que se van produciendo en la convivencia entre las personas y los grupos, así como el cambio producido en la economía en los últimos tiempo, generan nuevas demandas (GUILLEN, 1990), originando que un mercado en rápida transformación construya nuevas tipologías ocupacionales, como también puso de manifiesto, en España, el "Libro Blanco. Titulo de Grado en Pedagogía y Educación Social” (2004).

Brint (1994) diferencia entre profesiones técnicas, orientadas a la solución técnica de problemas y temas de la misma índole (ingenieros, arquitectos, informáticos etc.), aunque a posteriori sean las personas las que se beneficien de la tarea realizada por ellos, y las profesiones sociales, cuya finalidad es servir directamente a la ciudadanía. Las profesiones sociales, en los tiempos actuales, realizan su travesía particular hacia nuevos escenarios, y, para ello, precisan renovar sus motivaciones y sus prácticas, su ética y sus códigos de conducta. En su evolución se produce una fructífera geografía de modos de ser, de hacer y de convivir que se compendian en diversos campos profesionales.

Así, "con los términos "profesiones sociales” aludimos a un campo multiprofesional que está vertebrado en torno a la acción social, tiene en común el servicio a las personas” (GARCÍA ROCA, 2000, p. 313).

Se pregunta García Roca (2000, p. 313) por el sentido en el que pueden ser sociales unas profesiones, $y$, para él, el adjetivo social es solo una nota constituida de actividad humana, no alude únicamente al carácter de los problemas a los que responde, ya que resultan sociales más bien porque son tratados por ciertas profesiones; "lo social se refiere a que todas ellas producen sociabilidad, crean y generan vínculos sociales, promueven la comunicación interhumana, activan la cooperación entre ciudadanos en situacio- 
nes asimétricas, generan relaciones e interacciones y mantienen vivo el tejido social".

El proceso de profesionalización, de las profesiones sociales, se relaciona con la idea de que la finalidad de las acciones profesionales es el servicio directo a las personas, en particular y, en general, a la ciudadanía. Porque en la lógica de las profesiones sociales palpita el impulso de lo vivo, y su secreto es la capacidad de crear significados y vincularse a los seres singulares, a los contextos vitales: actuar es siempre actuar con otras personas y el conocimiento social está cruzado por la pasión (GARCÍA ROCA, 2001), dinamizando la relación y vinculación con los sujetos en sus travesías vitales, así como el proceso de acompañamiento para que cada persona pueda tomar las riendas de su vida, porque "la educación es la antifatalidad, no el acomodo programado a ella el ideal básico que la educación actual debe conservar y promocionar es la universalidad democrática. Universalizar la educación consiste en terminar con las discriminaciones (SAVATER, 1997, p. 154).

A continuación aportamos algunas cuestiones iniciales sobre dos ámbitos históricos de la Pedagogía Social y de la Educación Social: la educación a lo largo de toda la vida, y la acción educativa de educadores y educadoras sociales en drogodependencias.

\section{LA EDUCACIÓN A LO LARGO DE TODA LA VIDA}

La educación de personas adultas mayores es uno de los ámbitos abordado desde la Pedagogía Social y temática abordada desde la Sociedad Iberoamericana de Pedagogía Social (SIPS) en la celebración de diversos eventos.

En el marco de la Educación Permanente, los Organismos Internacionales recomiendan un aprendizaje continuo, a lo largo de la vida, con el fin de orientar a los individuos hacia el perfeccionamiento de su ser, en un contexto determinado, a fin de propiciar el despliegue de posibilidades en sus dimen- siones, personal, profesional y social. Aprender a lo largo de la vida se ha convertido en una exigencia de la sociedad actual con el fin de participar en los diferentes foros sociales y evitar la exclusión social. Como indica Ríos (2007, p. 295) “la educación permanente se ha convertido en la herramienta más necesaria para luchar contra las desigualdades, las injusticias y las situaciones que privan a las personas de vivir libre y dignamente en su medio".

El Informe de la Organización de las Naciones Unidas para la Educación, la Ciencia y la Cultura UNESCO (1996) de la Comisión Internacional sobre la Educación para el siglo XXI, presidida por Jacques Delors "La educación encierra un tesoro", sostiene que una de las claves indispensables para el desarrollo de los pueblos es la educación y la formación a lo largo de la vida. La experiencia ha demostrado que la educación contribuye a lograr la equidad, la igualdad social y la no discriminación.

La educación para todos y en todas las edades constituye un principio fundamental en las políticas educativas. Pueden citarse muchos ejemplos en este sentido. La Unión Europea ha promovido múltiples iniciativas entre las que destaca la publicación del Memorándum sobre el Aprendizaje Permanente (2000), que está llamado a convertirse en el principio rector de la oferta de servicios a los ciudadanos. Igualmente, dedicó el año 2007 a promover la igualdad de oportunidades y la no discriminación por razones de edad, sexo y condición social. La educación continua permitirá a todos adquirir los conocimientos necesarios para participar como ciudadanos de pleno derecho en la sociedad del conocimiento y la información, por medio de las tecnologías de la información y la comunicación.

En el plano internacional son numerosos los documentos originados en distintos foros que defienden esta misma postura. Entre ellos cabe destacar la Conferencia Mundial de Derechos Humanos (1993), la Conferencia Internacional sobre la Población y el 
Desarrollo (1994), la Cumbre Mundial sobre Desarrollo Social (1995), el Foro Mundial sobre la Educación para Todos (2000) y la VI Conferencia Internacional sobre la Educación de Adultos (2009). Todos han dado como resultado la adopción de compromisos para actuar en esta línea. Los gobiernos asumieron la obligación de velar para que se alcancen y refuercen los objetivos y finalidades de la educación para todos. Una educación orientada a desarrollar los talentos y capacidades de cada sujeto con el fin de mejorar su calidad de vida.

El Plan de Acción Internacional Madrid 2002 sobre el Envejecimiento aprobado en la II Asamblea Mundial del Envejecimiento, propone como objetivos los siguientes: ofrecer oportunidades al individuo de desarrollo, de realización personal y de bienestar, la posibilidad de acceder al aprendizaje y la necesidad de reconocer la contribución social, cultural, económica y política de las personas de edad. Se insiste pues en propiciar el acceso al conocimiento, la educación y la capacitación de las personas mayores, como base indispensable para disponer de una vida activa que garantice su enriquecimiento a lo largo de toda su existencia. Por todas estas razones, las personas mayores son una "apuesta" de futuro.

El acceso a los bienes culturales y a las posibilidades formativas de este sector es responsabilidad de todos, por lo que se hace necesario un esfuerzo compartido de las distintas administraciones, en tanto que gestionan los recursos sociales y educativos y de las Universidades como centros de promoción del saber. En España se han producido importantes avances tal como se refleja en el informe sobre las personas mayores, publicado por el Instituto de Mayores y Servicios Sociales (IMSERSO, 2006), cuya fecha coincide con la aprobación de la Ley de Promoción de la Autonomía Personal y Atención a las Personas en situación de Dependencia en el Congreso de los Diputados (30 de noviembre). Ha sido este un momento histórico para la ciudadanía española sin diferencia de edad, ya que sus consecuencias y marco protector afectan a todos, tal como se ha señalado con anterioridad.
Existe la creencia errónea, de que la persona al hacerse mayor va perdiendo el interés por aprender cosas nuevas. Se ha demostrado que no es correcto el modelo del déficit, basado en una perspectiva médica tradicional, que establece que la persona va perdiendo con los años el deseo por aprender cosas nuevas. Cada vez son más las que viven su vejez desde el modelo de desarrollo llegando a la edad de jubilación con ganas de seguir formándose y con ello mejorar su vida.

\section{REFERENCIAS}

ASEDES. El educador y la educadora social en el Estado español: una concreción de su trabajo en centros escolares. 2007. Disponible en: <www.eduso.net/archivos/ESCE.doc>. Acceso en: 10/07/2013.

BARRAYCOA MARTÍNEZ, J. LASAGA MILLET, O. La importancia de las competencias instrumentales de la empleabilidad: El caso de los conocimientos informáticos. 2010. Disponible en: <http//www. uao.es/investigación-1/copy_of:observatorio-laboral/investigación/ artículos>. Acceso en: 17/07/2013

BAS-PEÑA, E., CAMPILLO DÍAZ, M. SÁEZ CARRERAS, J. La educación social: Universidad, estado y profesión. Barcelona: Laertes, 2010.

BAS-PEÑA, E., PÉREZ-DE-GUZMÁN, V. Y VARGAS-VERGARA, M. Educación y Género: La formación de los educadores y educadoras sociales. Pedagogía Social. Revista Interuniversitaria, 23, 2014, p.95-120. DOI:10.7179/PSRI_2014.23.05.

BRINT, S. In an Age of Experts: The Changing Role of Professions in Politics and Public Life. Princeton, NJ: Princeton University Press, 1994.

CARIDE, J.A. Las fronteras de la Pedagogía Social. Perspectivas científica e histórica. Barcelona: Gedisa. Educación Social, 2005.

DELORS, J. La Educación encierra un tesoro. Madrid: 
UNESCO, 1996.

FERMOSO, P. Historia de la Pedagogía Social española. Valencia: NauLlibres, 2003.

FLEXNER, A. Is Social Work a Profession? Studies in Social Work, 4, 1915.

FREIDSON, E. The futures of professionalization. In: M. STACEY et al. (eds.), Health and the division of labour. Londres, Croom Helm, 1977, p.14-38.

FREIDSON, E. La teoría de las profesiones: estado del arte. Perfiles Educativos, vol. XXIII, n. 93, p.28-43. Universidad Nacional Autónoma de México. México, D.F., México, 2001.

GARCÍA ROCA, J. La navegación y la fisonomía del naufragio: KISNERMAN, N (Comp.). Etica ¿un discurso o una práctica social? Buenos Aires, Argentina: Paidós, 2001.

GARCÍA ROCA, J. Trabajo Social. In: CORTINA, A. Y CONILL, J. 10 palabras clave en ética de las profesiones. Buenos Aires, Argentina: Verbo Divino, 2000.

GINER DE LOS RÍOS, F. La Universidad española, Obras completas. Madrid: Espasa Calpe, 1916.

GUILLEN, M. F. Profesionales y burocracia: desprofesionalización, proletarización y poder profesional en las organizaciones complejas. REIS: Revista Española de Investigaciones Sociológicas, (51), 1990, p.35-51.

JOHNSON, T. GERRY LARKIN. MIKE SAKS (eds.) Health Professions and the State in Europe. London: Routledge, 1995.

KEMMIS, S. MCTAGGART, R. Cómo planificar la Investigación-Acción. Barcelona: Laertes, 1992.

LARSON, M.S. The Rise of Professionalism. Berkeley, University of California, 1977.
LARSON, M.S. In the Matter of Experts and Professionals, or How Impossible It Is to Leave Nothing Unsaid. In: TORSTENDAH, R. BURRAGE, M. (Eds.) The Formation of Professional Knowledge. State and Strategy, London SAGE Publications, 1990.

MORIN, E. Introducción al pensamiento complejo. Barcelona: Gedisa, 1998.

MORIN, E. Los siete saberes necesarios para la educación del futuro. Barcelona: Paidós; Moyano, 2001.

NACIONES UNIDAS. Declaración Política y Plan de Acción Internacional de Madrid sobre el Envejecimiento. Nueva York: Naciones Unidas, 2003.

NUÑEZ, V. Pedagogía social: Cartas para navegar en el nuevo milenio. Buenos Aires: Santillana, 1999.

ONU. Conferencia Mundial de Derechos Humanos. Viena: Naciones Unidas, 1993.

ONU. Conferencia Internacional sobre la Población y el Desarrollo (1994). Nueva York: Naciones Unidas, 1995.

ONU. Cumbre Mundial sobre Desarrollo Social. Copenhague, Dinamarca: Naciones Unidas, 1995.

ORTEGA y GASSET, J. Obras completas, v.IV. Misión de la universidad. Madrid: Revista de Occidente, 1930, pp.531-562.

ORTIZ, L. F. Campus Virtual: la educación más allá del LMS. Revista de Universidad y Sociedad del Conocimiento (RUSC), 4, 1, 2007, p.25-46.

PANAIA, M. Una revisión de la sociología de las profesiones desde la teoría crítica del trabajo en la Argentina. Comisión Económica para América Latina y el Caribe (CEPAL). Naciones Unidas. Colección Documentos de proyectos, 2008. 
PÉREZ SERRANO, G. Pedagogía social-educación social: construcción científica e intervención práctica. Madrid: Narcea, 2003.

PETRUS, A. Concepto y campos de la educación social. Actas del Congreso sobre Educación Social en España. Madrid: CIDE, 1989.

QUINTANA, J. M. La profesionalización de la Pedagogía Social. Pedagogía Social. Revista Interuniversitaria, 1, 1986, p.39-45.

QUINTANA, J. M. Pedagogía Social. Madrid: Dykinson, 1984.

RIOS, M. Tendencias del aprendizaje permanente en el Espacio Europeo. Cuestiones Pedagógicas, 18, 2007, p.271-284.

SÁEZ, J. La pedagogía social en España: sugerencias para la reflexión. Revista de Pedagogía Social, n.1, 1986, p.7-21.
SÁEZ, J. Pedagogía Social y Educación Social. Historia, profesión y competencias. Madrid: Pearson, 2007.

SAVATER, F. El valor de educar. Barcelona: Ariel, 1997.

TORSTENDAHL, R. La transformación de la educación profesional en el siglo XIX. En Rothblatt, Sh. y Wittrock, B. (coords.) La Universidad Europea y americana desde 1800. Las tres transformaciones de la Universidad. Barcelona: Pomares, 1996, p.147-148.

UNESCO. Foro Mundial sobre la Educación para Todos. Dakar: UNESCO, 2000.

UNESCO. Sexta Conferencia Internacional sobre la Educación de Adultos. Brasil: UNESCO, 2009.

VITERI DÍAZ, G. Política Social: elementos para su discusión, Edición electrónica gratuita. 2007. Disponible en: <www.eumed.net/libros/2007b/297/>. Acceso en: 10/07/2013. 